\title{
Propiedades psicométricas del «Cuestionario para la Evaluación del Síndrome de Quemarse por el Trabajo» (CESQT) en profesionales de la salud italianos: una perspectiva de género
}

\author{
Psychometric properties of the «Spanish Burnout Inventory» (SBI) in a sample \\ of Italian health professionals: a gender perspective
}

\author{
Pedro R. Gil-Monte ${ }^{\mathrm{a},{ }^{*}}$, Sara Viotti ${ }^{\mathrm{b}}$, Daniela Converso ${ }^{\mathrm{b}}$ \\ ânidad de Investigación Psicosocial de la Conducta Organizacional (UNIPSICO), Universitat de València, España \\ bUniversidad de Turín, Departamento de Psicología, Italia
}

\section{Resumen}

El objetivo de este estudio fue analizar las propiedades psicométricas del Cuestionario para la Evaluación del Síndrome de Quemarse por el Trabajo (CESQT) en una muestra de profesionales de la salud italianos. La muestra estuvo formada por 695 participantes (453 profesionales de enfermería y 242 médicos). Según el género se identificaron 451 mujeres (64.9\%) y 244 hombres (35.1\%). El CESQT está formado por 20 ítems que se distribuyen en cuatro dimensiones: Ilusión por el trabajo (5 ítems), Desgaste psíquico (4 ítems), Indolencia (6 ítems), y Culpa (5 ítems). La estructura factorial se analizó mediante análisis factorial confirmatorio. Todos los ítems presentaron valores adecuados para los parámetros estadísticos y psicométricos, al igual que las cuatro escalas. El modelo de 4 factores que reproduce la estructura original del cuestionario presentó un ajuste adecuado a los datos, con invarianza factorial entre la muestra de hombres y la muestra de mujeres. Se concluye que el CESQT tiene propiedades psicométricas adecuadas para el estudio del síndrome de quemarse por el trabajo (burnout) en profesionales de la salud italianos con independencia del género de esos profesionales.

Palabras clave: síndrome de quemarse por el trabajo, CESQT, género, análisis factorial confirmatorio, invarianza factorial, profesionales de la salud.

\section{Para citar este artículo:}

Gil-Monte, P. R., Viotti, S., \& Converso, D. (2017). Propiedades psicométricas del «Cuestionario para la Evaluación del Síndrome de Quemarse por el Trabajo» (CESQT) en profesionales de la salud italianos: una perspectiva de género. Liberabit, 23(2), 153-168. doi: 10.24265/liberabit.2017.v23n2.01

\begin{abstract}
The purpose of this study was to assess the psychometric properties of the Spanish Burnout Inventory (SBI) in a sample of Italian health professionals. The sample consisted of 695 participants: 453 nursing professionals and 242 physicians. Regarding the gender, 451 were women (64.9\%) and 244 were men (35.1\%). The SBI is composed of 20 items distributed in four dimensions: Enthusiasm towards the job (5 items), Psychological exhaustion (4 items), Indolence (6 items) and Guilt (5 items). The factor structure was examined through confirmatory factor analysis. All items achieved appropriate values for both statistical and psychometric parameters, just as the four scales did. The four-factor model consistent with the Spanish original obtained a good data fit for the whole sample, showing factor invariance in both samples (i.e., men vs. women). In conclusion, the SBI has adequate psychometric properties to study the burnout syndrome in Italian health professionals, regardless of their gender.
\end{abstract}

Keywords: burnout, Spanish Burnout Inventory, SBI, gender, confirmatory factor analysis, factor invariance, health professionals.

Este es un artículo Open Access bajo la licencia Creative Commons Atribución-NoComercial-CompartirIgual 4.0

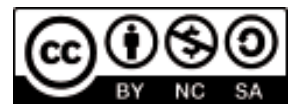




\section{Introducción}

Los trabajadores del sector ocupacional de la salud son una población con alto riesgo de experimentar niveles elevados de estrés laboral crónico y problemas derivados de esta exposición, como el deterioro de la calidad de vida (Grimaldo, 2011). Entre esos problemas se encuentra el síndrome de quemarse por el trabajo (burnout) (en adelante SQT) que contribuye al deterioro de su calidad de vida laboral (Suñer-Soler et al., 2013).

El creciente interés por las condiciones de trabajo del personal sanitario (Lamiani, Borghi, \& Argentero, 2015) apunta a que el estrés laboral y el SQT tienen como consecuencia un incremento en el número de errores médicos (Rama-Maceiras, \& Kranke, 2013). Por otra parte, el estrés laboral está generalizado entre los profesionales de enfermería (Happell et al., 2013), y este colectivo presenta con frecuencia niveles elevados de estrés laboral. Algunos estudios sobre SQT han concluido que entre los médicos el porcentaje de SQT puede estar entre el 30\% y el 40\% (Wallace, Lemaire, \& Ghali, 2009), o incluso puede ser superior en USA (Kumar, 2016; Shanafelt et al., 2012) y en países de Latinoamérica (Castañeda \& García, 2010). Klersy et al. (2007) en un estudio con médicos y enfermeras en Italia concluyeron que la prevalencia del SQT en profesionales de enfermería estaba en el $30 \%$, y en el $18 \%$ en el caso de los médicos.

El SQT es una respuesta al estrés laboral crónico. Es una experiencia subjetiva de carácter negativo compuesta por cogniciones, emociones y actitudes negativas hacia el trabajo, hacia las personas con las que se relaciona el individuo en su trabajo, en especial los clientes, y hacia el propio rol profesional. Como consecuencia de esta respuesta aparecen una serie de disfunciones conductuales, psicológicas y fisiológicas que van a tener repercusiones nocivas para las personas y para la organización (Gil-Monte, 2011).
La definición más extendida sobre el SQT es la elaborada por Maslach y Jackson (1981), quienes lo definieron como un síndrome cuyos síntomas son: (a) baja realización personal en el trabajo, entendida como la tendencia de los profesionales a evaluar negativamente su actividad laboral y el ejercicio de la misma, (b) agotamiento emocional, caracterizado por la experiencia de no poder dar más de sí mismos a nivel afectivo, y (c) despersonalización, definida como el desarrollo de actitudes y sentimientos negativos hacia las personas destinatarias del trabajo.

El Maslach Burnout Inventory (MBI) (Maslach \& Jackson, 1981) es el cuestionario más utilizado para evaluar el SQT. Sus tres dimensiones se ajustan a la definición anterior (Maslach, Schaufeli, \& Leiter, 2001). Sin embargo, este instrumento presenta algunos problemas psicométricos (Olivares-Faúndez \& Gil-Monte, 2009; Wheeler, Vassar, Worley, \& Barnes, 2011). Entre esos problemas se citan los siguientes: (a) algunos estudios han concluido que se deberían considerar solo dos factores en lugar de los tres que propone el modelo, con los ítems de las escalas de Agotamiento emocional y Despersonalización formando un factor (lo que se ha llamado el «núcleo del burnout») y el segundo factor formado por los ítems de la escala de Realización personal (Holland, Michael, \& Kim, 1994; Walkey \& Green, 1992); (b) la dimensión de Despersonalización presenta con demasiada frecuencia valores de consistencia interna demasiado bajos (Chao, McCallion, \& Nickle, 2011), y (c) debería evaluar de manera más amplia el SQT, pues no lo recoge en toda su amplitud. Según Halbesleben y Demerouti (2005) la dimensión de Agotamiento emocional debería incorporar otros aspectos de agotamiento, como agotamiento cognitivo y físico, para recoger de una manera más completa la experiencia de agotamiento que experimentan los trabajadores como consecuencia de su exposición al estrés laboral crónico.

Una definición alternativa sobre el SQT ha sido elaborada por Gil-Monte (2005). Según este autor, el 
SQT es una respuesta psicológica al estrés laboral crónico de carácter interpersonal y emocional que aparece en los profesionales de las organizaciones de servicios que trabajan en contacto con los clientes o usuarios de la organización. Esta respuesta se caracteriza por cuatro síntomas: (a) un deterioro cognitivo (pérdida de la ilusión por el trabajo o baja realización personal en el trabajo), (b) por un deterioro afectivo (agotamiento emocional y físico), (c) por la aparición de actitudes y conductas negativas hacia los clientes y hacia la organización, en forma de comportamientos indiferentes, fríos, distantes y, en ocasiones, lesivos (indolencia) y, en algunos casos, (d) estos síntomas se acompañan de sentimientos de culpa.

Para evaluar esos síntomas se desarrolló el «Cuestionario para la Evaluación del Síndrome de Quemarse por el Trabajo» (CESQT) ${ }^{1}$ (Gil-Monte, 2011; Gil-Monte \& Olivares-Faúndez, 2011). El CESQT está basado en un modelo según el cual el deterioro cognitivo (bajas puntuaciones en Ilusión por el trabajo) y el deterioro afectivo (altas puntuaciones en Desgaste psíquico) son las primeras manifestaciones del SQT como respuesta al estrés laboral crónico. A estos síntomas le sigue la aparición de actitudes negativas hacia las personas con las que se trabaja (altas puntuaciones en Indolencia). Además, algunos trabajadores, no todos, desarrollan altos sentimientos de culpa debido a estos síntomas, en especial por el trato desconsiderado e indolente hacia los clientes o usuarios de los servicios (GilMonte, 2005). Los sentimientos de culpa han sido identificados por diferentes autores como un síntoma del SQT (Burisch, 2006; Maslach, 1982; Price \& Murphy, 1984).

Desde que se iniciaron las investigaciones para explicar el proceso de desarrollo del SQT (Paine, 1982) hasta la actualidad (van Dam, 2016) son varios los estudios que vienen recomendado la necesidad de diferenciar entre tipos o grupos de individuos con SQT (Farber, 2000; Gil-Monte, 2012; Tops et al., 2007; Vanheule, Lievrouw, \& Verhaeghe, 2003) pues, aunque los profesionales que desarrollan esta patología comparten la mayoría de los síntomas, se observa que no en todos tiene las mismas consecuencias, o que su intensidad varía. Además, diferenciar perfiles en el desarrollo del SQT pueden ayudar a diferenciarlo de otras patologías con las que comparte síntomas, como la depresión (Ahola \& Hakanen, 2007; Bianchi, Boffy, Hingray, Truchot, \& Laurent, 2013; Bianchi, Schonfeld, \& Laurent, 2015; Melamed, Shirom, Toker, Berliner, \& Shapira, 2006; Tement, Pahor, \& Jaušovec, 2016; Toker \& Biron, 2012).

Tomando como referencia el modelo teórico desde el que se ha construido el cuestionario, la evaluación con el CESQT permite diferenciar dos perfiles de individuos en el desarrollo del SQT incorporando la variable "Sentimientos de culpa», y ayuda a diferenciarlo de otros problemas de salud y sus consecuencias (Gil-Monte, 2012). El Perfil 1 conduce a la aparición de un conjunto de sentimientos y conductas vinculados al estrés laboral que originan una forma moderada de malestar, pero que no incapacita al individuo para el ejercicio de su trabajo, aunque podría realizarlo mejor. Este perfil se caracteriza por la presencia de baja ilusión por el trabajo, junto a altos niveles de desgaste psíquico e indolencia, pero los individuos no presentan niveles críticos de sentimientos de culpa, pues la indolencia funciona como estrategia de afrontamiento funcional para manejar el estrés laboral y sus síntomas. El Perfil 2 constituye con frecuencia un problema más serio que identificaría a los individuos más deteriorados por el desarrollo del SQT. Además de los síntomas anteriores, los individuos presentan niveles más altos de sentimientos de culpa. 
El CESQT tiene algunas ventajas sobre otros instrumentos utilizados en la evaluación del SQT. Entre esas ventajas están: (a) es un instrumento basado en un modelo teórico con un desarrollo previo al modelo psicométrico y que sustenta a éste (GilMonte, 2005), (b) aunque alguna de sus dimensiones son similares a las del MBI, el CESQT incorpora la evaluación de los sentimientos de culpa y dos perfiles de SQT, además incluye ítems para evaluar aspectos físicos del agotamiento y no solo aspectos psicológicos (Gil-Monte, 2011), (c) no presenta insuficiencias psicométricas cuando se aplica en idiomas diferentes al español (Bosle \& Gil-Monte, 2010; FigueiredoFerraz, Gil-Monte, Queirós, \& Passos, 2014; Misiolek, Gil-Monte, \& Misiolek, 2016; Viotti, Gil-Monte, \& Converso, 2015).

Los resultados obtenidos en estudios previos sobre las características psicométricas del CESQT han respaldado la estructura de cuatro factores mediante análisis factorial exploratorio y análisis factorial confirmatorio, y han obtenido resultados adecuados de consistencia interna, mediante el índice alfa de Cronbach, para las cuatro subescalas. Estos resultados se han obtenido en estudios con profesionales de la salud realizados en España (GilMonte \& Manzano-García, 2015), Italia (Viotti et al., 2015), México (Gil-Monte \& Zúñiga-Caballero, 2010), y Polonia (Misiolek et al., 2016). También se ha obtenido valores adecuados para la validez concurrente entre el CESQT y el MBI. Los valores de correlación para las escalas de estos dos cuestionarios van de .35 a .52 para la correlación entre Ilusión por el trabajo y Realización personal en el trabajo, de .68 a .77 para la correlación entre Desgaste psíquico y Agotamiento emocional, y de .39 a .52 para la correlación entre Indolencia y Despersonalización (Gil-Monte \& Olivares-Faúndez, 2011; Viotti et al., 2015), e incluso valores superiores en estudios menos recientes. Estos valores se pueden considerar moderados y aceptables (Cohen, 1988), lo que implica que el constructo teórico del SQT estimado mediante el CESQT es similar al de otras escalas aceptadas y utilizadas internacionalmente.
El género es una variable sociodemográfica que explica diferencias significativas en los niveles del SQT (Purvanova \& Muros, 2010). Aunque en algún estudio previo realizado con profesionales de enfermería italianos (Viotti et al., 2015) se ha obtenido evidencia empírica para una estructura de cuatro factores en el CESQT mediante análisis factorial exploratorio, en la actualidad no existen estudios factoriales realizados mediante análisis factorial confirmatorio, ni que hayan considerado la validez transversal de cuestionario según el género, en muestras italianas.

Por ello el objetivo de este estudio es analizar la validez factorial del modelo psicométrico del CESQT y su invarianza de género en una muestra italiana de profesionales de la salud para contribuir a la validez transnacional del cuestionario en italiano. Sobre la base de los resultados obtenidos en estudios previos sobre la estructura factorial del instrumento, se hipotetiza que el modelo de cuatro factores obtendrá un ajuste adecuado a los datos.

\section{Método}

\section{Participantes}

La muestra estuvo compuesta por 695 profesionales de la salud, de los que 242 (34.80\%) fueron médicos y 453 (65.20\%) profesionales de enfermería. Según el género de los participantes 244 (35.10\%) fueron hombres y 451 (64.90\%) fueron mujeres. Considerando ambas variables de manera conjunta, en el grupo de hombres se contabilizaron 146 médicos y 98 profesionales de enfermería (21\% y $14.10 \%$ respectivamente sobre el total de la muestra), mientras que en el grupo de mujeres se contabilizaron 96 médicos y 355 profesionales de enfermería (13.80\% y 51.10\% respectivamente sobre el total de la muestra). La prueba $\chi^{2}$ para la distribución de casos por grupos indicó que existían diferencias significativas en la distribución de los participantes en los subgrupos generados por el cruce de ambas variables $\left(\chi_{(1)}^{2}=103.68, p<.001\right)$. 
La media de edad para el total de la muestra fue $M=45.42$ años $(D E=9.33$, mín. = 24, máx. = 67). La media para la antigüedad laboral fue $\mathrm{M}=17.98$ años $(D E=10.20$, mín. = 1, máx. $=44)$.

\section{Instrumento}

Los participantes respondieron a la adaptación al italiano (Viotti et al., 2015) del «Cuestionario para la Evaluación del Síndrome de Quemarse por el Trabajo» (CESQT) (Gil-Monte, 2011). Este instrumento se compone de 20 ítems distribuidos en cuatro dimensiones denominadas: (a) Ilusión por el trabajo: deseo del individuo de alcanzar las metas laborales, ya que suponen una fuente de placer personal, 5 ítems, (p.e., «Me siento ilusionado/a por mi trabajo»), (b) Desgaste psíquico: agotamiento emocional y físico debido a que, en el trabajo, se tiene que tratar a diario con personas que presentan o causan problemas, 4 ítems, (p.e., "Me siento desgastado/a emocionalmente»), (c) Indolencia: actitudes negativas de indiferencia y cinismo hacia los «clientes de la organización» 6 ítems, (p.e., «Pienso que trato con indiferencia a algunos pacientes»), y (d) Culpa: sentimientos de culpa por las actitudes negativas desarrolladas en el trabajo, en especial con las personas hacia las que se trabaja, 5 ítems, (p.e., «Me siento culpable por alguna de mis actitudes en el trabajo»). Los ítems se responden mediante una escala de frecuencia de cinco grados (0 «Nunca» a 4 «Muy frecuentemente: todos los días»).

Bajas puntuaciones en Ilusión por el trabajo junto con altas puntuaciones en Desgaste psíquico e Indolencia, además de altas puntuaciones en Culpa, indican altos niveles de SQT. Adicionalmente, el CESQT permite diferenciar dos perfiles en el desarrollo del SQT. Para obtener estos perfiles se combina la puntuación resultado del promedio de los 15 ítems de las escalas de Ilusión por el trabajo (invertida), Desgaste psíquico e Indolencia, en combinación con la puntuación obtenida en la escala de Culpa (Misiolek et al., 2016).

\section{Procedimiento}

La recogida de datos se realizó en la zona sanitaria de Turín. Los participantes trabajaban en atención primaria, dos hospitales con varias especialidades médico-quirúrgicas, y un hospital especializado en el diagnóstico y tratamiento de enfermedades infecciosas. Antes de iniciar la recogida de datos se obtuvo la aprobación de la dirección de los centros de trabajo, que revisaron el plan de investigación, los instrumentos y método de recogida de datos, y su utilización con fines científicos. Se consideró como población para el estudio a todos los trabajadores cuya categoría profesional fuese de medicina o de enfermería. Los datos fueron recogidos en el Servicio de Medicina del Trabajo como parte de la revisión médica anual que se realiza a los trabajadores. La participación en el estudio fue voluntaria y no aleatoria. Todos los participantes firmaron un documento de consentimiento informado por el que aceptaban responder al cuestionario de forma anónima, en cumplimiento de la ley italiana sobre privacidad de datos. Después de eliminar los casos no válidos, la tasa de respuesta sobre la población considerada para el estudio fue del $68 \%$.

\section{Análisis de datos}

Para estimar los estadísticos descriptivos (media y desviación típica), consistencia interna y correlaciones ( $r$ de Pearson) entre las variables se utilizó el programa SPSS 22. La omega de McDonald (1970) se estimó mediante el programa R versión 3.3.3 (Dunn, Baguley, \& Brunsden, 2014). Para probar los modelos factoriales se utilizó análisis factorial confirmatorio con el programa AMOS 22. El método de estimación fue máxima verosimilitud. Se sugiere la utilización de varios índices para probar el ajuste del modelo a los datos (Byrne, 2001). Debido a la sensibilidad del estadístico $\chi^{2}$ al tamaño de la muestra se han utilizado otros índices de ajuste: $\chi^{2} /$ gl (valores inferiores a 3 se consideran indicadores de ajuste aceptable del modelo) (Carmines \& McIver, 1981; Kline, 2005). Para el GFI (Goodness of Fit Index), el NNFI (Non 
Normed Fit Index), y el CFI (Comparative Fit Index) algunos autores han recomendado valores superiores a .90 (Kline, 2005) como indicadores de un buen ajuste del modelo. Para el RMSEA (Root Mean Square Error of Approximation) valores entre .05 y .08 indican un ajuste adecuado del modelo (Hair, Anderson, Tatham, \& Black, 1995), y en el caso del índice SRMR (Standardized Root Mean Squared Residual) valores inferiores a .08 se consideran indicadores de un buen ajuste ( $\mathrm{Hu} \&$ Bentler, 1999).

La evaluación de la invarianza se realizó siguiendo un patrón de modelos analizados en los que se van añadiendo restricciones al aumentar el número de parámetros que se comparan entre los grupos hasta que se rechaza un modelo. Para concluir sobre el nivel de invarianza se comparó el ajuste de los modelos anidados mediante la diferencia entre los valores del CFI. Si el valor de la diferencia entre dos modelos anidados es superior a .01 en favor del modelo menos restrictivo se debe rechazar el modelo con más restricciones (Cheung \& Rensvold, 2002). Entonces se analizan los valores de los parámetros para identificar los que dan lugar a la no invarianza del modelo.

Se diseñaron cinco modelos, el análisis se inició con la evaluación del modelo de: (1) invarianza configural (misma estructura factorial en ambos grupos), para pasar a probar la (2) invarianza métrica o invarianza débil (incorpora la igualdad de cargas factoriales), (3) invarianza escalar o invarianza fuerte (incorpora la igualdad de los interceptos) (4) invarianza residual o invarianza estricta (incorpora la igualdad de la varianza de error de las variables observadas). Con estos análisis se prueba la invarianza de medida. Además, se probó la invarianza estructural. Para ello se incorporó (5) la invarianza de las varianza y de las covarianzas de los factores (Byrne, 2008; Dimitrov, 2010).

\section{Resultados}

Para alcanzar el objetivo del estudio se realizaron tres tipos de análisis de datos: (1) análisis descriptivo de los ítems, (2) evaluación de la estructura factorial del CESQT, y (3) análisis de la consistencia interna de las escalas.

\section{Análisis descriptivo de los ítems}

La Tabla 1 presenta los estadísticos descriptivos para las escalas y para los ítems del CESQT, la correlación entre el ítem y la escala, y el valor alfa de Cronbach de la escala cuando se elimina el ítem de ella. El ítem con el valor más alto para la media fue el ítem 10 (Pienso que mi trabajo me aporta cosas positivas), tanto para la muestra de hombres $(\mathrm{M}=2.64)$ como para la de mujeres $(\mathrm{M}=2.75)$ que pertenece a la escala de Ilusión por el trabajo. Con respecto al ítem con el valor más bajo, en la muestra de hombres fue el ítem 20 (Me siento mal por algunas cosas que he dicho en el trabajo) ( $\mathrm{M}=$ 0.61) que pertenece a la escala de Culpa, y en la muestra de mujeres el ítem 11 (Me apetece ser irónico/a con algunos pacientes) que pertenece a la escala de Indolencia. De los 20 ítems del cuestionario sólo en la muestra de hombres dos ítems excedieron ligeramente el rango \pm 1 para el valor de la asimetría. Estos ítems fueron el ítem 7 (As = 1.11) y el ítem 11 (As = 1.36). Los dos ítems pertenecen a la escala de Indolencia.

\section{Análisis de la estructura factorial}

Para probar los modelos se estableció como modelo hipotetizado de partida el modelo de 4 factores validado en el manual del cuestionario (Gil-Monte, 2011). Este modelo establece una estructura de cuatro factores descrita en la sección Instrumento y fue validado previamente en italiano (Viotti et al., 2015). El modelo de cuatro factores se evaluó para toda la muestra y para los subgrupos generados por la variable género (hombres vs. mujeres). 


\section{Tabla 1}

Estadísticos descriptivos para los ítems del CESQT.

\begin{tabular}{|c|c|c|c|c|}
\hline $\begin{array}{l}\text { Escala } \\
\text { Ítem }\end{array}$ & $M(d t)$ & $\begin{array}{c}\text { Correlación } \\
\text { ítem-escala } \\
\text { corregida }\end{array}$ & Asimetría & $\begin{array}{c}\text { Alfa si se } \\
\text { elimina } \\
\text { ítem }\end{array}$ \\
\hline & $\mathrm{H} / \mathrm{M}$ & $\mathrm{H} / \mathrm{M}$ & $\mathrm{H} / \mathrm{M}$ & $\mathrm{H} / \mathrm{M}$ \\
\hline Ilusión por el trabajo & 2.54(.74) / 2.61(.67) & & & \\
\hline 1. Trabajo reto & 2.61(.81) / 2.52(.82) & $.70 / .60$ & $-.24 /-.44$ & $.88 / .87$ \\
\hline 5. Realización personal & $2.54(.91) / 2.60(.88)$ & $.70 / .65$ & $-.38 /-.53$ & $.88 / .86$ \\
\hline 10. Cosas positivas & 2.64(.82 / 2.75(.74) & $.75 / .73$ & $-.19 /-.37$ & $.87 / .84$ \\
\hline 15. Trabajo gratificante & $2.60(.85) / 2.70(.80)$ & $.80 / .78$ & $-.41 /-.57$ & $.86 / .82$ \\
\hline 19. Ilusión & $2.32(.98) / 2.50(.89)$ & $.76 / .74$ & $-.34 /-.44$ & $.87 / .83$ \\
\hline Desgaste psíquico & $1.60(.80) / 1.77(.79)$ & & & \\
\hline 8. Saturado/a & $1.47(1.02) / 1.60(.97)$ & $.65 / .66$ & $.19 / .15$ & $.81 / .83$ \\
\hline 12. Agobiado/a & $1.79(.93) / 1.86(.94)$ & $.69 / .70$ & $-.04 / .01$ & $.80 / .81$ \\
\hline 17. Cansancio físico & $1.85(.94) / 2.07(.90)$ & $.71 / .71$ & $.09 / .03$ & $.79 / .81$ \\
\hline 18. Desgaste emocional & $1.30(.98) / 1.56(.98)$ & $.66 / .70$ & $.47 / .28$ & $.81 / .81$ \\
\hline Indolencia & $1.24(.63) / 1.17(.55)$ & & & \\
\hline 2. No atender & $1.38(.95) / 1.33(.82)$ & $.50 / .56$ & $.33 / .10$ & $.74 / .70$ \\
\hline 3. Insoportables & 1.37(.89) / 1.33(.82) & $.65 / .59$ & $.34 / .38$ & $.70 / .69$ \\
\hline 6. Pesados & 2.15(1.01)/ 2.11(.91) & $.42 / .34$ & $.03 /-.01$ & $.76 / .77$ \\
\hline 7. Indiferencia & $.75(.85) / .67(.76)$ & $.57 / .52$ & $1.11 / 1.00$ & $.72 / .71$ \\
\hline 11. Ironía & $.69(.89) / .64(.75)$ & $.48 / .44$ & $1.36 / .87$ & $.74 / .73$ \\
\hline 14. Etiquetar & $1.09(.95) / .94(.85)$ & $.49 / .54$ & $.49 / .48$ & $.74 / .71$ \\
\hline Culpa & $.92(.61) / 1.01(.61)$ & & & \\
\hline 4. Preocupa trato & $1.53(1.05) / 1.54(1.05)$ & $.48 / .46$ & $.29 / .25$ & $.85 / .83$ \\
\hline 9. Culpa actitudes & $.84(.74) / 1.01(.76)$ & $.68 / .68$ & $.38 / .34$ & $.76 / .74$ \\
\hline 13. Remordimientos & $.74(.70) / .76(.75)$ & $.75 / .68$ & $.42 / .64$ & $.75 / .74$ \\
\hline 16. Pedir disculpas & $.88(.75) / .92(.73)$ & $.63 / .61$ & $.56 / .40$ & $.78 / .76$ \\
\hline 20. Mal cosas dichas & $.61(.69) / .80(.72)$ & $.62 / .60$ & $.86 / .40$ & $.78 / .77$ \\
\hline
\end{tabular}

Nota 1. El número del ítem indica su posición en el cuestionario.

Nota 2. EL CESQT se aplicó en italiano.

Nota 3. H, valores para la muestra de hombres $(n=244)$. M, valores para la muestra de mujeres $(n=451)$. 
El modelo obtuvo un buen ajuste a los datos en los dos subgrupos de la variable género (ver Tabla 2). En la muestra de hombres $(n=244)$ los valores de los índices de ajuste global fueron: $\chi_{(164)}^{2}=282.80$, $p<.001$, RMSEA $=.055$, intervalo de confianza $90 \%$ (CIs) [.044 - .065], SRMR $=.060$, GFI $=.893$, NNFI $=.933, \mathrm{y} \mathrm{CFI}=.943$. Y en la muestra de mujeres $(\mathrm{n}$ $=451): \chi_{(164)}^{2}=413.07, p<.001$, RMSEA $=.058$, intervalo de confianza 90\% (CIs) [.051 - .065],
SRMR $=.057$, GFI $=.916$, NNFI $=.920$, y CFI $=$ .931. Sólo en la muestra de hombres el valor del índice GFI resultó inferior a .90. Todas las cargas factoriales resultaron significativas para $p<.001$ en las dos muestras. El valor más bajo para la relación ítemfactor se obtuvo en las dos muestras para el ítem 6 que carga en el factor Indolencia. En el caso de la muestra de hombres ese valor fue $\lambda=.47$, y en la muestra de mujeres alcanzó un valor de $\lambda=.37$.

Tabla 2

Índices de ajuste de los modelos para el CESQT

\begin{tabular}{lcccccccc}
\hline Modelo & $\chi^{2}$ & $\mathrm{gl}$ & $\chi^{2} / \mathrm{gl}$ & RMSEA $_{(90 \% \mathrm{Cl})}$ & SRMR & GFI & NNFI & CFI \\
\hline $\begin{array}{l}\text { Hombres } \\
(\mathrm{n}=244)\end{array}$ & 282.80 & 164 & 1.72 & $.055_{(.044-065)}$ & .060 & .893 & .933 & .943 \\
$\begin{array}{l}\text { Mujeres } \\
(\mathrm{n}=451)\end{array}$ & 413.07 & 164 & 2.53 & $.058_{(.051-065)}$ & .057 & .916 & .920 & .931 \\
$\begin{array}{l}\text { Muestra total } \\
(\mathrm{n}=695)\end{array}$ & 490.78 & 164 & 2.99 & $.054_{(.048-0.059)}$ & .052 & .933 & .932 & .942 \\
\hline
\end{tabular}

Nota 1. $\chi^{2}=$ chi-cuadrado, gl = grados de libertad, RMSEA $_{(\mathrm{Cl})}=$ Root Mean Square Error of Approximation (90\% confidence intervals), SRMR = Standardized Root Mean Squared Residual, GFI = Goodness-of-Fit Index, NNFI = Non-Normed Fit Index, $\mathrm{CFI}=$ Comparative Fit Index.

Nota 2. Para todos los valores de chi-cuadrado, $p<.001$.

No se obtuvo evidencia sobre la existencia de cargas cruzadas para ningún ítem, ni en la muestra de hombres ni en la muestra de mujeres. Según los índices de modificación, en las dos muestras el valor más alto para la liberación de relaciones factor-ítem se obtendría al liberar la relación entre el ítem 4 asignado a la escala de Culpa y el factor Ilusión por el trabajo (hombres, $\lambda=.26$, IM $=7.21$; mujeres, $\lambda=.09$, $\mathrm{IM}=$ 15.67).

Para probar si la estructura factorial resultaba equivalente entre la muestra de hombres y mujeres se siguió el procedimiento de modelos anidados descrito en la sección Procedimiento. El modelo configural presentó un buen ajuste a los datos (Tabla 3): RMSEA $=.040$, intervalo de confianza $90 \%$ (CIs) [.036 - .044], CFI $\left.=.935, \chi^{2} / g\right]=2.12$, lo que indica que la estructura factorial es adecuada para las dos muestras (hombres vs. mujeres).
Dado que los resultados apoyaron la invarianza configural del modelo se probó la invarianza métrica (invarianza débil). Esto es, la intensidad entre las relaciones de los ítems con sus respectivas escalas es similar en las dos muestras. El valor de la diferencia entre los índices CFI del modelo configural y del modelo métrico $(\Delta \mathrm{CFI}=.001)$ resultó inferior a .01, lo que sustenta este tipo de invarianza. También resultó inferior a .01 la diferencia entre los valores del CFI del modelo métrico y del modelo escalar $(\Delta \mathrm{CFI}=.008)$, $\mathrm{y}$ entre el modelo escalar $\mathrm{y}$ el modelo residual $(\Delta \mathrm{CFI}=.005)$. Estos resultados apoyan la invarianza fuerte y la invarianza estricta, respectivamente, por lo que el modelo factorial presente invarianza de medida.

Un paso más para probar la invarianza entre los grupos consiste en el análisis de la invarianza estructural (igualdad de varianza y convarianzas de 
Propiedades psicométricas del «Cuestionario para la Evaluación del Síndrome de Quemarse por el Trabajo» (CESQT) en profesionales de la salud italianos: una perspectiva de género

Tabla 3

Índices de ajuste para la prueba de invarianza del modelo según el sexo de los participantes

\begin{tabular}{lcccccc}
\hline Modelo & $\chi^{2}$ & $\mathrm{gl}$ & $\chi^{2} / \mathrm{gl}$ & RMSEA $_{(90 \% \mathrm{Cl})}$ & SRMR & CFI \\
\hline 1. Configural & 695.94 & 328 & 2.12 & $.040_{(.036-044)}$ & .060 & .935 \\
2. Métrico & 704.96 & 344 & 2.05 & $.039_{(.035-043)}$ & .061 & .936 \\
3. Escalar & 774.14 & 364 & 2.13 & $.040_{(.036-044)}$ & .061 & .928 \\
4. Residual & 820.69 & 384 & 2.14 & $.041_{(.037-044)}$ & .062 & .923 \\
5. Estructural & 846.13 & 394 & 2.15 & $.041_{(.037-044)}$ & .073 & .920 \\
\hline
\end{tabular}

Nota 1. $\chi^{2}=$ chi-cuadrado, $\mathrm{gl}=$ grados de libertad, $\mathrm{RMSEA}_{(\mathrm{Cl})}=$ Root Mean Square Error of Approximation (90\% confidence intervals), SRMR $=$ Standardized Root Mean Squared Residual, CFI = Comparative Fit Index.

Nota 2. Para todos los valores de chi-cuadrado, $p<.001$.

los constructos). Para ello se analizó la diferencia en los valores del CFI entre el modelo residual y el modelo estructural. La diferencia entre los valores del CFI obtuvo un valor de .003 lo que sustenta la invarianza estructural del modelo factorial.

El modelo también obtuvo un ajuste adecuado para el total de la muestra $(n=695)$ (Tabla 2): $\chi_{(164)}^{2}$ $=$ 490.78, $p<.001$, RMSEA $=.054$, intervalo de confianza 90\% (CIs) [.048 - .059], GFI = .933, NNFI $=.932$, CFI $=.942$. Todas las cargas factoriales resultaron significativas, y las covarianzas para las relaciones entre las dimensiones del CESQT también resultaron significativas para $p<.001$, excepto la relación entre Ilusión por el trabajo y Culpa (cov $=$ $.005, p=.722$ ). Todas las relaciones ítem-factor resultaron significativas. El valor más bajo se obtuvo para la relación entre el ítem 6 (Creo que los familiares de los pacientes son unos pesados) y el factor de Indolencia. El valor del parámetro para está relación fue $\lambda=.41$. Los índices de modificación no indicaron la existencia de cargas cruzadas (crossloadings). Caso de ser liberado, la relación de cualquier ítem con un factor diferente al que estaba asignado según el modelo psicométrico propuesto no superaba en ningún caso el valor de |.10|.

Adicionalmente se realizaron análisis de diferencias de medias para los ítems considerando la variable género. Sólo se obtuvieron diferencias de medias para seis ítems. Tres de la escala de Culpa (ítems, 9, 14 y 20), dos de la escala de Desgaste psíquico (ítems 17 y 18), y uno de la escala de Ilusión por el trabajo (ítem 19). En dos ítems de la escala de Culpa la media para la muestra de mujeres fue significativamente más alta que la media para la muestra de hombres (ítem 9, $\mathrm{M}_{\mathrm{H}}$ $=0.84$ vs. $\mathrm{M}_{\mathrm{M}}=1.01, t=-2.81, p<.01$; ítem $14, \mathrm{M}_{\mathrm{H}}$ $=1.09$ vs. $\mathrm{M}_{\mathrm{M}}=0.94, t=2.06, p<.05$; ítem $20, \mathrm{M}_{\mathrm{H}}$ $=0.61$ vs. $\left.\mathrm{M}_{\mathrm{M}}=0.80, t=-3.41, p<.001\right)$. En los dos ítems de la escala de Desgaste psíquico la media para la muestra de mujeres fue significativamente más alta que la media para la muestra de hombres (ítem 17, $\mathrm{M}_{\mathrm{H}}=1.85$ vs. $\mathrm{M}_{\mathrm{M}}=2.07, t=-2.93, p<.01$; ítem 18, $\mathrm{M}_{\mathrm{H}}=1.09$ vs. $\left.\mathrm{M}_{\mathrm{M}}=0.94, t=-3.39, p<.001\right)$. Y para el ítem de la escala de Ilusión por el trabajo la media para la muestra de hombres resultó significativamente más baja que la media para la muestra de mujeres (ítem 19, $\mathrm{M}_{\mathrm{H}}=2.32$ vs. $\mathrm{M}_{\mathrm{M}}=2.50, t=-2.43, p<.05$ ).

Para las escalas no se obtuvieron diferencias significativas entre los valores de la media en las escalas de Ilusión por el trabajo $\left(\mathrm{M}_{\mathrm{H}}=2.54\right.$ vs. $\mathrm{M}_{\mathrm{M}}$ $=2.61, t=-1.26, p>.05)$, Indolencia $\left(\mathrm{M}_{\mathrm{H}}=1.60 \mathrm{vs}\right.$. $\left.\mathrm{M}_{\mathrm{M}}=1.77, t=1.43, p>.05\right)$ y Culpa $\left(\mathrm{M}_{\mathrm{H}}=0.92\right.$ vs. $\left.\mathrm{M}_{\mathrm{M}}=1.01, t=-1.81, p>.05\right)$. Las diferencias resultaron significativas para la diferencia de medias en la escala de Desgaste psíquico $\left(\mathrm{M}_{\mathrm{H}}=1.60\right.$ vs. $\mathrm{M}_{\mathrm{M}}$ $=1.77, t=-2.68, \mathrm{p}<.01)$. 


\section{Análisis de las escalas}

La Tabla 4 presenta los estadísticos descriptivos para las escalas del CESQT, y las correlaciones entre ellas. Las puntuaciones para todas las escalas se ajustaron a una distribución normal en la muestra de hombres y en la muestra de mujeres, pues presentaron valores de asimetría y de curtosis dentro del rango \pm 1 . El valor del alfa de Cronbach alcanzó valores mayores de .70 para las dos muestras según el género: Ilusión por le trabajo $\left(\alpha_{\mathrm{H}}=.90, \alpha_{\mathrm{M}}=.87\right)$, Desgaste psíquico $\left(\alpha_{\mathrm{H}}=.85, \alpha_{\mathrm{M}}=.85\right)$, Indolencia $\left(\alpha_{\mathrm{H}}=.77, \alpha_{\mathrm{M}}=.76\right)$, y Culpa $\left(\alpha_{\mathrm{H}}=.84, \alpha_{\mathrm{M}}=.82\right)$ (Tabla 4$)$.

Adicionalmente, para estimar la consistencia interna de las escalas en las dos muestras se calculó la omega de McDonald (1970). Este parámetro es la estimación complementaria más adecuada cuando el principio de tau equivalencia que se asume para calcular el alfa de Cronbach no se cumple, ya que en ese caso el alfa de Cronbach subestima la consistencia interna de las escalas (Dunn et al., 2014). Al igual que el alfa de Cronbach, valores del omega superiores a .70 indican que la consistencia interna de las escalas es aceptable. El omega alcanzó valores mayores de .70 en todas las escalas para las dos muestras: Ilusión por el trabajo $\left(\omega_{\mathrm{H}}=.90, \omega_{\mathrm{M}}=\right.$ $.87)$, Desgaste psíquico $\left(\omega_{\mathrm{H}}=.84, \omega_{\mathrm{M}}=.85\right)$, Indolencia $\left(\omega_{\mathrm{H}}=.77, \omega_{\mathrm{M}}=.76\right)$, y Culpa $\left(\omega_{\mathrm{H}}=.82\right.$, $\omega_{\mathrm{M}}=.80$ ) (Tabla 4).

\section{Tabla 4}

Estadísticos descriptivos para las dimensiones del CESQT y correlaciones $r$ de Pearson.

\begin{tabular}{|c|c|c|c|c|c|c|c|c|c|c|}
\hline & $\mathrm{M}(d t)$ & As & $\mathrm{Cu}$ & $\alpha$ & $\omega$ & Rango & 1 & 2 & 3 & 4 \\
\hline & $\mathrm{H}$ & $\mathrm{H}$ & $\mathrm{H}$ & $\mathrm{H}$ & $\mathrm{H}$ & & $\mathrm{H}$ & $\mathrm{H}$ & $\mathrm{H}$ & $\mathrm{H}$ \\
\hline & M & M & M & M & M & & M & M & M & M \\
\hline \multirow[t]{2}{*}{ 1. Ilusión por el trabajo } & $2.54(.74)$ & -.32 & .44 & $.90_{(.87-91)}$ & $.90_{(.87-92)}$ & \multirow[b]{2}{*}{$0-4$} & \multirow[b]{2}{*}{1} & \multirow[b]{2}{*}{$-.17 * *$} & \multirow[b]{2}{*}{$-.12^{*}$} & \multirow[b]{2}{*}{.11} \\
\hline & 2.61(.67) & -.45 & .59 & $.87_{(.84-89)}$ & $.87_{(.85-89)}$ & & & & & \\
\hline \multirow{2}{*}{ 2. Desgaste psíquico } & $1.60(.80)$ & .29 & .30 & $.85_{(.81-.87)}$ & $.85_{(.80-88)}$ & \multirow{2}{*}{$0-4$} & \multirow[b]{2}{*}{$-.35 * * *$} & \multirow[b]{2}{*}{1} & \multirow[b]{2}{*}{$.43^{* * *}$} & \multirow[b]{2}{*}{$* .28 * * *$} \\
\hline & $1.77(.79)$ & .33 & .20 & $.85_{(.83-87)}$ & $.85_{(.82-87)}$ & & & & & \\
\hline 3. Indolencia & $\begin{array}{l}1.24(.63) \\
1.17(.55)\end{array}$ & $\begin{array}{l}.74 \\
.36\end{array}$ & $\begin{array}{r}1.35 \\
.18\end{array}$ & $\begin{array}{l}.77_{(.72-.81)} \\
.76_{(.72-79)}\end{array}$ & $\begin{array}{l}.77_{(.70-82)} .76_{(.72-79)} \\
\text {. }\end{array}$ & $0-4$ & $-.19 * * *$ & $.29 * * *$ & 1 & $.43^{* * *}$ \\
\hline \multirow{2}{*}{ 4. Culpa } & $.92(.61)$ & .35 & -.64 & $.84_{(.78-85)}$ & $.82_{(.77-85)}$ & \multirow[b]{2}{*}{$0-4$} & \multirow[b]{2}{*}{.05} & \multirow[b]{2}{*}{$.31^{* * *}$} & & \multirow[b]{2}{*}{1} \\
\hline & $1.01(.61)$ & .32 & -.02 & $.82_{(.77-83)}$ & $.80_{(.77-83)}$ & & & & $.35^{* * *}$ & \\
\hline
\end{tabular}

$* p<.05, * * p<.01, * * * p<.001$

Nota 1. Los valores alfa de Cronbach y omega de McDonald se ofrecen con intervalos de confianza 95\%.

Nota 2. H, valores para la muestra de hombres. M, valores para la muestra de mujeres.

Nota 3. Los valores de las correlaciones sobre la diagonal corresponden a la muestra de hombres y los inferiores a la muestra de mu

Con respecto a la relación entre ítems y escalas, la correlación corregida entre el ítem y la escala presentó valores superiores a .40 para todos los ítems en las dos muestras, con excepción del ítem 6 en la muestra de mujeres $(r=.34)$ que pertenece a la escala de Indolencia (Tabla 1). Todos los ítems contribuyeron a incrementar o mantener el valor de consistencia interna de la escala a la que pertenecen, pues al eliminarlos disminuía el valor alfa de Cronbach de la escala. La única excepción la presentó el ítem 4 (Me preocupa el trato que he dado a algunas personas en el trabajo) que pertenece a la escala de Culpa. Su eliminación incrementó en un punto el valor de la consistencia interna de esta escala. Este 
resultado se obtuvo en la muestra de hombres y en la de mujeres (ver Tabla 1).

Para la relación entre las escalas se obtuvo que todos los valores para la correlación $r$ de Pearson estuvieron en la dirección esperada. Los valores resultaron significativos para $p<.05$ con excepción del valor para la correlación entre Ilusión por el trabajo y Culpa, tanto para la muestra de hombres $(r=.11, p>.05)$, como para la muestra de mujeres $(r=.05, p>.05)$. Además, se analizó la diferencia del valor de la correlación considerando el género. En dos casos el valor de la asociación entre variables resultó significativamente diferente en función del género. Así, la correlación entre Ilusión por el trabajo y Desgaste psíquico resultó significativamente menos intensa en la muestra de hombres que en la de mujeres $\left(r_{\mathrm{H}}=-.17\right.$ vs. $\left.r_{\mathrm{M}}=-.35, \mathrm{z}=2.43, p<.05\right)$, mientras que la correlación entre Desgaste psíquico e Indolencia resultó significativamente más intensa en la muestra de hombres que en la de mujeres $\left(r_{\mathrm{H}}=\right.$ .43 vs. $\left.r_{\mathrm{M}}=.29, z=2.02, p<.05\right)$.

\section{Discusión}

El objetivo de este estudio ha sido analizar la validez transnacional del CESQT y su invarianza de género en una muestra italiana de profesionales de la salud con el fin de concluir sobre la validez del cuestionario en un idioma y en una cultura diferente al que fue creado. Para alcanzar el objetivo del estudio se ha probado la validez psicométrica de los ítems que forman el cuestionario, su estructura factorial con invarianza de género y la consistencia interna de las cuatro escalas que lo forman.

Los resultados obtenidos en el análisis de asimetría de los ítems siguen el patrón de estudios previos realizados en profesionales de la salud con la versión española del cuestionario (Gil-Monte \& Manzano-García, 2015; Gil-Monte \& ZúñigaCaballero, 2010). El ítem 11 y el ítem 7 han presentado los valores más elevados de asimetría tanto en la muestra de hombres como en la de mujeres. Para todos los ítems, y en las dos submuestras del estudio (hombres vs. mujeres) los valores de asimetría estuvieron dentro del intervalo que se considera aceptable para concluir que la distribución de las puntuaciones ajusta a la distribución normal (Miles \& Shevlin, 2005).

Cuando se consideró el valor de la media de los ítems se observa que en la muestra de hombres y en la de mujeres el valor más alto y más bajo de la media coincide en los mismos ítems en las escalas de Ilusión por el trabajo (ítem 10 y 19, respectivamente), Desgaste psíquico (ítems 17 y 18, respectivamente), e Indolencia (ítems 6 y 11, respectivamente). En la escala de Culpa hubo coincidencia en el caso de la media más elevada (ítem 4), pero no en el de la más baja. Estos resultados son similares a los obtenidos en el estudio de Gil-Monte y Manzano-García (2015) y también, en el caso de los ítems con puntuaciones más bajas, al estudio de Gil-Monte y Zúñiga-Caballero (2010). La mayor disparidad en el caso de este segundo estudio puede deberse a la diferencia cultural dado que esta realizado en México.

Los valores para la correlación ítem-escala fueron relativamente altos para la mayoría de los ítems, lo que indica que tanto en la muestra de hombres como en la de mujeres las dimensiones del CESQT se puede considerar una función lineal de los ítems que contienen.

Las diferencias significativas entre las medias de los ítems según el género de los participantes fueron muy pocas, pues sólo se obtuvieron en el caso de 6 ítems, con una tendencia que indica que las mujeres tienden a puntuar significativamente más alto que los hombres en los síntomas de deterioro emocional. Este resultado está en línea con lo obtenido en los estudios sobre el SQT (Purvanova \& Muros, 2010), y sustentan la validez del CESQT en italiano para hombres y mujeres.

Considerando la estructura factorial del CESQT los resultados confirmaron la hipótesis formulada, pues el modelo de cuatro factores obtuvo un ajuste 
adecuado a los datos. Estos resultados permiten concluir que el modelo factorial del CESQT en su versión en italiano ajusta de manera adecuada al modelo teórico original (Gil-Monte, 2011), que incluye cuatro dimensiones o síntomas de SQT: Ilusión por el trabajo, Desgaste psíquico, Indolencia y Culpa.

Los resultados también permiten afirmar que se ha obtenido evidencia para la invarianza de medida (Elosua, 2005) y para la invarianza estructural (Byrne, 2008) del modelo de cuatro factores según el género, pues las diferencias en el índice CFI presentaron valores inferiores a .01 en todas las comparaciones (Cheung \& Rensvold, 2002). La invarianza de medida se refiere al grado en que las variables observadas o ítems del cuestionario presentan parámetros similares en los dos grupos, y se ha evaluado a tres niveles: débil (invarianza de las cargas factoriales), fuerte (invarianza de las cargas factoriales y de los interceptos) y estricta (invarianza de las cargas factoriales, de los interceptos y de las varianzas de los errores). Se ha obtenido evidencia de la invarianza para los tres niveles. La invarianza estructural se centra en la invarianza de las varianzas y covarianzas de los factores (variables latentes o no observadas) (Byrne, 2008; Dimitrov, 2010). Los resultados también apoyan este tipo de invarianza según el género para la muestra del estudio.

Los índices de consistencia interna de las cuatro escalas que forman el CESQT fueron adecuados, con valores alfa de Cronbach y omega de McDonald en el intervalo que va de .76 a .90 (Nunnally, 1978; McDonald, 1970). Las correlaciones entre las escalas resultaron significativas y en la dirección esperada con excepción de la correlación entre la escala de Ilusión por el trabajo y Culpa en la muestra de hombres y en la muestra de mujeres. Los valores de correlación $r$ de Pearson obtenidos en estudios previos para la relación entre estas dos escalas siempre han sido los más bajos del conjunto de relaciones entre las escalas, y se sitúan entre $r=-.03$ (Gil-Monte, FigueiredoFerraz, \& Valdez-Bonilla, 2013) y $r=-.35$ (FigueiredoFerraz et al., 2014), estando los valores más frecuentes entre $r=-.10$ y $r=-.15$. Sin embargo, en este estudio llama la atención el valor positivo de las correlaciones, pues lo esperable según el modelo teórico del CESQT (Gil-Monte, 2005) es que el valor de la correlación entre esa dos variables tenga un valor negativo. Un resultado similar sólo se ha obtenido en un estudio previo realizado con trabajadores chilenos de atención al público en una caja de compensación $(r=.11$, Olivares \& Gil-Monte, 2007). Este resultado es difícil de explicar teóricamente y, dado el valor y la dirección de la correlación entre estas dos variables con el resto de variables del estudio y los valores de otros parámetros estadísticos (e.g., media, dt, asimetría y curtosis) no parece que se deba a un sesgo estadístico. Una explicación que puede justificar el resultado obtenido para la correlación es que los profesionales más ilusionados por su trabajo se implicarían más en él y en el trato con los pacientes, por lo que siguiendo el modelo teórico del CESQT ello les llevaría desarrollar más sentimientos de culpa. De manera que, en estos profesionales los niveles más altos de ilusión por el trabajo en lugar de prevenir los sentimientos de culpa parece que los favorecen por su mayor implicación.

Los resultados del estudio permiten concluir que el CESQT tiene propiedades psicométricas adecuadas para el estudio del SQT en profesionales de la salud italianos, tanto en hombres como en mujeres. La relevancia del estudio es que contribuye a probar la validez psicométrica del cuestionario a nivel internacional con independencia del género de los profesionales, y se suma a otros estudios que ya han probado esas características psicométricas en idiomas diferentes al castellano. Así, pues se consolida la validez transcultural del CESQT.

Futuros estudios deben explorar la evidencia de puntos de corte con carácter de diagnóstico clínico, esto es, a partir de qué valor o de qué percentil los sujetos presentan niveles de SQT con una frecuencia tal que deteriora su salud con el fin de analizar el impacto epidemiológico real del SQT como enfermedad laboral. 


\section{Referencias}

Ahola, K., \& Hakanen, J. (2007). Job strain, burnout, and depressive symptoms: a prospective study among dentists. Journal of Affective Disorders, 104, 103-110. doi:10.1016/j.jad.2007.03.004

Bianchi, R., Boffy, C., Hingray, C., Truchot, D., \& Laurent, E. (2013). Comparative symptomatology of burnout and depression. Journal of Health Psychology, 18, 782-787. doi: $10.1177 / 1359105313481079$

Bianchi, R., Schonfeld, I. S., \& Laurent, E. (2015). Burnoutdepression overlap: a review. Clinical Psychology Review, 36, 28-41. doi: 10.1016/j.cpr.2015.01.004

Bosle, A., \& Gil-Monte, P. R. (2010). Psychometric properties of the Spanish Burnout Inventory in German professionals: preliminary results. Ansiedad y Estrés, 6(2-3), 283-291.

Burisch, M. (2006). Das Burnout-Syndrom: Theorie der inneren Erschöpfung [The burnout-syndrome: A theory of inner exhaustion]. Heidelberg: Springer Medizin Verlag.

Byrne, B. (2001). Structural equation modeling with Amos. London, UK: Lawrence Erlbaum Associates.

Byrne, B. (2008). Testing for multigroup equivalence of a measuring instrument: a walk through the process. Psicothema, 20, 872-882.

Carmines, E. G., \& McIver, J. P. (1981). Analyzing models with unobserved variables: Analysis of covariance structures. En G.W. Bornstedt y E. F. Borgatta (Eds.), Social measurement: Current issues (pp. 112-133). Beverly Hills, CA: Sage.

Castañeda, E., \& García, J. E. (2010). Prevalencia del síndrome de agotamiento profesional (burnout) en médicos familiares mexicanos: análisis de factores de riesgo. Revista Colombiana de Psiquiatría, 39, 67-84.

Chao, S. F., McCallion, P., \& Nickle, T. (2011). Factorial validity and consistency of the Maslach Burnout Inventory among staff working with persons with intellectual disability and dementia. Journal of Intellectual Disability Research, 55, 529-536. doi: 10.1111/j.1365-2788.2011.01413.x
Cheung, G. W., \& Rensvold, R. B. (2002). Evaluating goodness-of-fit indexes for testing measurement invariance. Structural Equation Modeling, 9, 233-255. doi: 10.1207/S15328007SEM0902_5

Cohen, J. (1988). Statistical power analysis for the behavioural sciences (2nd ed.). Hillsdale, NJ: Lawrence Erlbaum Associates.

Dunn, T. J., Baguley, T., \& Brunsden, V. (2014). From alpha to omega: A practical solution to the pervasive problem of internal consistency estimation. British Journal of Psychology, 105, 399-412. doi: 10.1111/bjop.12046

Dimitrov, D. M. (2010). Testing for factorial invariance in the context of construct validation. Measurement and Evaluation in Counseling and Development, 43, 121149. doi: 10.1177/0748175610373459

Elosua, P. (2005). Evaluación progresiva de la invarianza factorial entre las versiones original y adaptada de una escala de autoconcepto. Psicothema, 17, 356-362.

Farber, B. A. (2000). Treatment strategies for different types of teacher burnout. Journal of Clinical Psychology, 56, 675-689. doi: 10.1002/(SICI)1097-4679(200005)

Figueiredo-Ferraz, H., Gil-Monte, P. R., Queirós, C., \& Passos, F. (2014). Validação factorial do «Spanish Burnout Inventory» em polícias portugueses. Psicologia: Reflexao e Crítica, 27, 291-299.

Gil-Monte, P. R. (2005). El síndrome de quemarse por el trabajo (burnout). Una enfermedad laboral en la sociedad del bienestar. Madrid: Pirámide.

Gil-Monte, P. R. (2011). CESQT. Cuestionario para la Evaluación del Síndrome de Quemarse por el Trabajo. Manual. Madrid: TEA Ediciones.

Gil-Monte, P. R. (2012). Influence of guilt on the relationship between burnout and depression. European Psychologist, 17, 231-236. doi: 10.1027/10169040/a000096

Gil-Monte, P. R., Figueiredo-Ferraz, H., \& Valdez Bonilla, H. (2013). Factor analysis of the Spanish Burnout Inventory among Mexican prison employees. Canadian Journal of Behavioural Science, 45(2), 96104. doi: $10.1037 / \mathrm{a} 0027883$ 
Gil-Monte, P. R., \& Manzano-García, G. (2015). Psychometric properties of the Spanish Burnout Inventory among staff nurses. Journal of Psychiatric and Mental Health Nursing, 22, 756-763. doi: 10.1111/jpm.12255

Gil-Monte, P. R., \& Olivares-Faúndez, V. (2011). Psychometric properties of the «Spanish Burnout Inventory» in Chilean professionals working to physical disabled people. The Spanish Journal of Psychology, 14, 441-451. doi: 10.5209/rev_SJOP. 2011.v14.n1.40

Gil-Monte, P. R., \& Zúñiga-Caballero, L. C. (2010). Validez factorial del «Cuestionario para la Evaluación del Síndrome de Quemarse por el Trabajo» (CESQT) en una muestra de médicos mexicanos. Universitas Psychologica, 9, 169-178.

Grimaldo, M. (2011). Calidad de vida en profesionales de la salud en la ciudad de Lima. Liberabit, 17(2), 173185.

Hair, J. H., Anderson, R. E., Tatham, R. L., \& Black, W. C. (1995). Multivariate data analysis (4th ed.). Englewood Cliffs, NJ: Prentice-Hal.

Halbesleben, J. R., \& Demerouti, E. (2005). The construct validity of an alternative measure of burnout investigating the English translation of the Oldenburg Burnout Inventory. Work and Stress, 19, 208-220. doi: 10.1080/02678370500340728

Happell, B., Dwyer, T., Reid-Searl, K., Burke, K. J., Caperchione, C. M., \& Gaskin, C. J. (2013). Nurses and stress: recognizing causes and seeking solutions. Journal of Nursing Management, 21, 638-647. doi: 10.1111/jonm.12037

Holland, P. J., Michael, W. B., \& Kim, S. (1994). Construct validity of the Educators Survey for a sample of middle school teachers. Educational and Psychological Measurement, 54, 822-829. doi:10.1177/001316449405 4003029

Hu, L., \& Bentler, P.M. (1999). Cutoff criteria for fit indexes in covariance structure analysis: conventional criteria versus new alternatives. Structural Equation Modeling, 6, 1-55. doi: 10.1080/10705519909540118

Klersy, C., Callegari, A., Martinelli, V., Vizzardi, V., Navino, C., Malberti, F., ..., Politi, P. (2007). Burnout in health care providers of dialysis service in Northern Italy - a multicentre study. Nephrology Dialysis Transplantation, 22, 2283-2290. doi: 10.1093/ndt/gfm111

Kline, R. B. (2005). Principles and practice of structural equation modelling ( $2^{\mathrm{a}}$ ed.). New York: Guilford.

Kumar, S. (2016). Burnout and doctors: Prevalence, prevention and intervention. Healthcare, 4, 37. doi: 10.3 390/healthcare4030037

Lamiani, G., Borghi, L., \& Argentero, P. (2015). When healthcare professionals cannot do the right thing: A systematic review of moral distress and its correlates. Journal of Health Psychology, 22(1), 51-67. doi: 10.11 77/1359105315595120

Maslach, C. (1982). Burnout: The cost of caring. New York: Prentice Hall Press.

Maslach, C., \& Jackson, S. E. (1981). Maslach Burnout Inventory. Manual. Palo Alto, CA: Consulting Psychologists Press.

Maslach, C., Schaufeli, W. B., \& Leiter, M. P. (2001). Job burnout. Annual Review of Psychology, 52, 397-422. doi: 10.1146/annurev.psych.52.1.397

McDonald, R. P. (1970). Theoretical foundations of principal factor analysis and alpha factor analysis. British Journal of Mathematical and Statistical Psychology, 23, 1-21. doi: 10.1111/j.2044-8317.1970 .tb00432.x

Melamed, S., Shirom, A., Toker, S., Berliner, S., \& Shapira, I. (2006). Burnout and risk of cardiovascular disease: evidence, possible causal paths, and promising research directions. Psychological Bulletin, 132, 327353. doi: 10.1037/0033-2909.132.3.327

Miles, J., \& Shevlin, M. (2005). Applying regression \& correlation. A guide for students and researchers. London: Sage.

Misiolek, A., Gil-Monte, P. R., \& Misiolek, H. (2016). Prevalence of burnout in Polish anesthesiologists and anesthetist nursing professionals: A comparative nonrandomized cross-sectional study. Journal of Health Psychology, 22(4), 465-474. doi: 10.1177/135910531 5604377 
Nunnally, N. C. (1978). Psychometric Theory. New York: McGraw-Hill.

Olivares-Faúndez, V., \& Gil-Monte, P. R. (2009). Análisis de las principales fortalezas y debilidades del «Maslach Burnout Inventory»(MBI). Ciencia \& Trabajo, 11, 160-167.

Olivares, V. E., \& Gil-Monte, P. R. (2007). Análisis de las propiedades psicométricas del «Cuestionario para la Evaluación del Síndrome de Quemarse por el Trabajo» (CESQT) en profesionales chilenos. Ansiedad y Estrés, $13,229-240$.

Paine, W. S. (1982). The burnout syndrome in context. En J. W. Jones (Ed.), The burnout syndrome: Current research, theory, interventions (pp. 1-29). Park Ridge: London House Press.

Price, D. M., \& Murphy, P. A. (1984). Staff burnout in the perspective of grief theory. Death Education, 8, 4758. doi: 10.1080/07481188408251381

Purvanova, R. K., \& Muros, J. P. (2010). Gender differences in burnout: A meta-analysis. Journal of Vocational Behavior, 77, 168-185. doi: 10.1016/j.jvb.2010.04.006

Rama-Maceiras, P., \& Kranke, P. (2013) Working conditions and professional wellbeing: A link easy to imagine but difficult to prove. European Journal of Anesthesiology, 30, 213-215. doi: 10.1097/EJA.0b013 e32835fc894

Shanafelt, T. D., Boone, S., Tan, L., Dyrbye, L. N., Sotile, W., Satele, D., . . Oreskovich, M. R. (2012). Burnout and satisfaction with work-life balance among US physicians relative to the general US population. Archives of Internal Medicine, 172, 1377-1385. doi: 10.1 001/archinternmed.2012.3199

Suñer-Soler, R., Grau-Martín, A., Font-Mayolas, S., Gras, M. E., Bertrán, C., \& Sullman, M. J. (2013). Burnout and quality of life among Spanish healthcare personnel. Journal of Psychiatric and Mental Health Nursing, 20, 305-313. doi: 10.1111/j.1365-2850.2012.01897.x

Tement, S., Pahor, A., \& Jaušovec, N. (2016). EEG alpha frequency correlates of burnout and depression: The role of gender. Biological Psychology, 114, 1-12. doi: 10.1016/j.biopsycho.2015.11.005
Toker, S., \& Biron, M. (2012). Job burnout and depression: Unraveling their temporal relationship and considering the role of physical activity. Journal of Applied Psychology, 97, 699-710. doi: 10.1037/a0026914

Tops, M., Boksem, M. A., Wijers, A. A., van Duinen, H., Den Boer, J. A., Meijman, T. F., \& Korf, J. (2007). The psychobiology of burnout: Are there two different syndromes? Neuropsychobiology, 55, 143-150. doi: 10.1 159/000106056

van Dam, A. (2016). Subgroup analysis in burnout: Relations between fatigue, anxiety, and depression. Frontiers in Psychology, 7, 90. doi: 10.3389/fpsyg.201 6.00090

Vanheule, S., Lievrouw, A., \& Verhaeghe, P. (2003). Burnout and intersubjectivity: A psychoanalytical study from a Lacanian perspective. Human Relations, 56, 321-328. doi: 10.1177/0018726703056003614

Viotti, S., Gil-Monte, P. R., \& Converso, D. (2015). Toward validating the Italian version of the «Spanish Burnout Inventory»: a preliminary study. Revista da Escola de Enfermagem da USP, 49, 819-825. doi: 10.1590/S0080623420150000500016

Walkey, F. H., \& Green, D. E. (1992). An exhaustive examination of the replicable factor structure of the Maslach Burnout Inventory. Educational and Psychological Measurement, 52, 309-323. doi:10.1177/ 0013164492052002006

Wallace, J. E., Lemaire, J. B., \& Ghali, W. A. (2009). Physician wellness: a missing quality indicator. Lancet, 374, 1714-1721. doi: 10.1016/S0140-6736(09)61424-0

Wheeler D. L., Vassar M., Worley J. A., \& Barnes, L. L. (2011). A reliability generalization meta-analysis of coefficient Alpha for the Maslach Burnout Inventory. Educational and Psychological Measurement, 71, 231-244. doi: 10.1177/0013164410391579 


\section{Pedro R. Gil-Monte}

Universitat de València

Catedrático de Psicología Social y de las Organizaciones en la Universitat de València (España). Sus líneas de investigación se encuadran en la Psicología de la Salud Ocupacional y la calidad de vida laboral (v.g., burnout, violencia en el trabajo, conciliación trabajo-familia, salud laboral...).

Autor corresponsal: Pedro.Gil-Monte@uv.es

\section{Sara Viotti}

Università degli Studi di Torino

PhD en Psicología de la Salud y Calidad de Vida en 2011. En la actualidad trabaja como investigadora en el Departamento de Psicología de la Università degli Studi di Torino (Italia). Sus intereses de investigación principales son: burnout, competencias en el trabajo y violencia en el lugar de trabajo.

sara.viotti@gmail.com

\section{Daniela Converso}

Università degli Studi di Torino

Profesora Titular (Associate Professor) de Psicología de la Salud Ocupacional y Psicología de la Salud en el Departamento de Psicología de la Università degli Studi di Torino (Italia). Sus intereses de investigación son calidad de vida laboral, estrés laboral, burnout, envejecimiento de la población laboral activa y empleo sostenible.

daniela.converso@unito.it

Agradecimientos. La participación de Pedro R. Gil-Monte en este estudio ha contado con el apoyo del proyecto de investigación Ref.: PSI2013-48185-R, Ministerio de Economía y Competitividad (MINECO) (Gobierno de España). 